\title{
EPIDEMIOLOGICAL PROFILE OF PATIENTS USING JANUS KINASE INHIBITORS IN A TERTIARY CENTER
}

Stan Richard Medeiros de Souza1,^, Marina de Azevedo Martins ${ }^{1}$, Lucas Guimarães Nolasco Farias ${ }^{1}$, José Victor Martinez², Renata Maria Monteiro Pinto ${ }^{1}$, Adriana Cristiane Machado ${ }^{1}$, Roberta de Almeida Pernambuco ${ }^{1}$, Sônia Maria Alvarenga Anti Loduca Lima ${ }^{1}$, Lucas Victória de Oliveira Martins ${ }^{1}$, Nathália de Carvalho Sacilotto ${ }^{1}$, Rina Dalva Neubarth Giorgi ${ }^{1}$

1.Instituto de Assistência Médica ao Servidor Público Estadual, São Paulo (SP), Brazil; 2.Pontifícia Universidade Católica de São Paulo, São Paulo (SP), Brazil.

*Corresponding author: stanrichardms@gmail.com

\section{BACKGROUND}

Janus kinase inhibitors (JAKi) are small molecules that block the JAK family enzymes, that play a role in cell-signaling processes leading to inflammation and immune responses observed in rheumatoid arthritis (RA). Three JAKi (tofacitinib, baricitinib and upadacitinib) have been approved for use in RA. In insufficient response to methotrexate within 3 to 6 months in patients with poor prognostic factors, any biologic disease-modifying antirheumatic drug (bDMARD) or JAKi should be added to the conventional synthetic DMARD (csDMARD). If this fails, any other biologic (from another or the same class) or targeted synthetic DMARD (tsDMARD) is recommended. The aim of the study is to compare the clinical profile and therapeutic response of the use of JAKi initiated in patients naive to biological treatment or biological switched.

\section{METHODS}

Retrospective study carried out through review of medical records of patients with RA using JAKi therapy in a tertiary center. Clinical and laboratory characteristics were analyzed and, subsequently, 2 groups were made: JAKi patients naive to biological and biological switched.

\section{RESULTS}

Sixty-one patients were retrieved through medical records, of which 93.5\% are female, with mean age of 57.8 years (standard deviation, SD $=9.83$ ). The mean duration disease was 14.18 years ( $S D=10.31$ ), with $88.5 \%$ patients presenting positivity for rheumatoid factor and/or ACPA. Ten percent of the total population were active smokers and $16 \%$ were former smokers. The mean score obtained in the Charlson comorbidity index was 2.11, representing $82 \%$ of the average survival in 10 years. There were only two major adverse events: one case of viral meningoencephalitis and one case of severe Tinea corporis, both with no need for drug withdrawal. Comparing the biological naïve and biological switched group, no differences were found in age, disease duration, comorbidity index, smoking history, CDAI at JAKi initiation, adverse effects and medication discontinuation rates. Biological naïve group had a higher frequency of low disease activity/remission (82.6\% vs. 56.6\%, $p=0.03$ ), less need for corticosteroid therapy (44.4\% vs. 76.4\%, $p=0.01$ ) and lower current mean CDAI (5.57 vs. 11.39, $p=0.02$ ).

\section{CONCLUSION}

Janus kinase inhibitors are capable of inducing remission or low disease activity in most patients if used immediately after cSDMARD or bDMARD. In biological switched patients, a greater difficulty in reaching the therapeutic target was seen.

\section{KEYWORDS}

Janus kinase inhibitor, Rheumatoid arthritis, Clinical profile. 